\title{
Corrigendum: Real-World Lenvatinib Versus Sorafenib in Patients With Advanced Hepatocellular Carcinoma: A Propensity Score Matching Analysis
}

\author{
Yuan-Hung Kuo ${ }^{1}$, Sheng-Nan Lu ${ }^{1}$, Yen-Yang Chen ${ }^{2}$, Kwong-Ming Kee ${ }^{1}$, Yi-Hao Yen ${ }^{1}$, \\ Chao-Hung Hung ${ }^{1}$, Tsung-Hui Hu ${ }^{1}$, Chien-Hung Chen ${ }^{1}$ and Jing-Houng Wang ${ }^{1 *}$ \\ ${ }^{1}$ Division of Hepatogastroenterology, Department of Internal Medicine, Kaohsiung Chang Gung Memorial Hospital and \\ Chang Gung University College of Medicine, Kaohsiung, Taiwan, ${ }^{2}$ Division of Hematology-Oncology, Department of Internal \\ Medicine, Kaohsiung Chang Gung Memorial Hospital and Chang Gung University College of Medicine, Kaohsiung, Taiwan
}

\section{OPEN ACCESS}

Approved by:

Frontiers Editorial Office,

Frontiers Media SA, Switzerland

${ }^{*}$ Correspondence:

Jing-Houng Wang

jinghoung2001@yahoo.com.tw

Specialty section:

This article was submitted to Gastrointestinal Cancers: Hepato

Pancreatic Biliary Cancers,

a section of the journal

Frontiers in Oncology

Received: 28 November 2021 Accepted: 01 December 2021 Published: 14 December 2021

Citation:

Kuo Y-H, Lu S-N, Chen Y-Y, Kee K-M, Yen $Y-H$, Hung $C-H$, Hu T-H,

Chen C-H and Wang J-H (2021)

Corrigendum: Real-World Lenvatinib

Versus Sorafenib in Patients With Advanced Hepatocellular Carcinoma: A Propensity Score Matching Analysis.

Front. Oncol. 11:823960.

doi: 10.3389/fonc.2021.823960
Keywords: hepatocellular carcinoma, lenvatinib, propensity score (PS) matching (PSM), sorafenib, progressionfree survival

\section{A Corrigendum on}

Real-World Lenvatinib Versus Sorafenib in Patients With Advanced Hepatocellular Carcinoma: A Propensity Score Matching Analysis

By Kuo Y-H, Lu S-N, Chen Y-Y, Kee K-M, Yen Y-H, Hung C-H, Hu T-H, Chen C-H and Wang J-H (2021) Front. Oncol. 11:737767. doi: 10.3389/fonc.2021.737767

In the original article, there was an error. The IRB Number of our article, "IRB No: 202100961B0", was typed mistakenly as "IRB No: 20210096B0".

A correction has been made to the last sentence of the Materials and Methods, Patients and the Ethics Statement".

The authors apologize for this error and state that this does not change the scientific conclusions of the article in any way. The original article has been updated.

Publisher's Note: All claims expressed in this article are solely those of the authors and do not necessarily represent those of their affiliated organizations, or those of the publisher, the editors and the reviewers. Any product that may be evaluated in this article, or claim that may be made by its manufacturer, is not guaranteed or endorsed by the publisher.

Copyright $\odot 2021 \mathrm{Kuo}, \mathrm{Lu}$, Chen, Kee, Yen, Hung, Hu, Chen and Wang. This is an open-access article distributed under the terms of the Creative Commons Attribution License (CC BY). The use, distribution or reproduction in other forums is permitted, provided the original author(s) and the copyright owner(s) are credited and that the original publication in this journal is cited, in accordance with accepted academic practice. No use, distribution or reproduction is permitted which does not comply with these terms. 